\title{
Some Relations Between Price, Expenses and Profit
}

\author{
Prof. PhD Rosen Nikolaev \\ University of Economics - Varna, Varna, Bulgaria \\ nikolaev_rosen@ue-varna.bg
}

\begin{abstract}
In the economical literature and also in practice two methods for determining the profit exist. The first one is with respect to the revenue (profitability in terms of revenue/price) and the second one is with respect to the expenses (profitability in terms of expenses). In the present paper the author makes an attempt to prove that it is an advisable and adequate approach to calculate the profit in terms of revenue/price. In parallel the relation between the changes in pricing and changes different components of determining the total expenses is examined.
\end{abstract}

Keywords: price/revenue, expenses, profit.

JEL Code: D24, E64; doi:10.36997/IJUSV-ESS/2019.8.2.3

\section{Въведение}

Идеята за тематиката на това изследване бе продиктувана от една реакция на производител, предизвикана от увеличението на цената на промишления ток. Действително през 2018 година на три пъти бе увеличавана цената на електрическата енергия, използвана в бизнеса. Подчертавам на три пъти бе увеличавана цената, а не три пъти е увеличена цената. Каква беше реакцията на собственик на пекарна, цитирам: „От разходите ми за “производство на един хляб, $10 \%$ са електроенергия. В момента цената на един хляб е 1,30 лв. и за да си покрия новите разходи трябва да увелича цената на 1,70 лв.“ Вярно ли е това? Разбира се, че не! Ето какви са неговите разсъждения: токът е увеличен три пъти. До момента $10 \%$ от досегашната цената 1,30 лв., т.е. 0,13 лв., са разходите за ток. Следователно $3^{*} 0,13=0,39 \approx 0,40$ лв. са новите разходи за електроенергия и $1,30+0,40=1,70$ лв. Кои са грешките при този начин на мислене:

1. Цената на електроенергията се е увеличила три пъти.

2. Дори увеличението да е три пьти, то по неговите разсъждения новите разходи са не 0,39 лв., а 0,26 лв. и тогава цената би била 1,56 лв., а не 1,70 лв.

3. Ако съответният производител има далновидно мислене и иска да запази пазарния си дял, то той би увеличил цената на ниво, на което да бъдат покрити новите разходи, но печалбата му в абсолютни единици (не в \%) да се запази в същия размер.

Целта, която си поставя авторьт на настоящото изложение е да бъде предложен обобщен адекватен модел за промяна цената на дадена стока, предизвикана, поради изменение на различни разходи, свързани с нейното производство, складиране и транспортиране.

\section{1. Определяне цена на основата на известни общи разходи и печалба}

На студенти в ОКС „Магистър“, които са с бакалавърска степен по икономика задавам следния въпрос: Общите разходи за производството на единица от дадена стока са 10 лв. Ако желаната печалба е 60\%, то на каква цена трябва да бъде продадена стоката?

Резултатът е: Около 30\% не могат да отговорят, а останалите 70\% отговарят 16 лв. и нито един не дава верния отговор.

Реакцията ми е следната: просто казано, печалбата е това, което, след като го прибера от приходите, това което остане е достатъчно, за да направя нови разходи за същото количество стока, от което са тези приходи. Нека вземем 60\% от 16 лв., което е 9,60 лв. Тогава остават 6,40 лв., които не са достатъчно, за да произведа новата единица от тази 
стока. Пьрво, печалбата е 6 лв., но тя не съответства на това, че тя е 60\%, а в действителност е $37,5 \%\left(\frac{6}{16} .100\right)$ и второ, при тази цена (16 лв.), действително е направена $60 \%$ надценка, а не че печалбата е $60 \%$.

Нека сега изведем формула, която определя цената, така че печалбата действително да е $60 \%$. Нека цената на стоката е $P$, разходите за производството й са $C$ и желаната печалба е $k_{P} \%$ ( $C$ и $k_{P} \%$ са известни, а търсим $P$ ). В своя публикация Иванов (Иванов, 2016, стр. $143)$ определя подхода като един от класическите, отчитащ някой от пазарните сили, използвайки термина „реверсивна калкулация“ - от цена към разходи.

$$
\begin{aligned}
& P=C+\frac{k_{P}}{100} . P \\
& P .\left(1-\frac{k_{P}}{100}\right)=C \\
& P=\frac{C}{\left(1-\frac{k_{P}}{100}\right)}
\end{aligned}
$$

Тогава в примера:

$$
P=\frac{10}{1-\frac{60}{100}}=\frac{10}{0,4}=\frac{100}{4}=25 \text { лв. }
$$

Действително, 60\% от прихода от 25 лв. са 15 лв. и остават отново 10 лв., които се използват за производството на нова единица от стоката. Тук е мястото да кажем в какви граници може да се изменя процентьт $k_{P}$ на печалбата и процента $l$ на надценката.

$k_{P} \in[0 ; 100]$, като $k_{P}=0$, ако $P=C$, а $k_{P}=100$, само ако $C=0$, т.е. целият приход е печалба, ако разходите са 0 .

$l \in[-100 ; \infty)$, като -100 е, ако имаме разходи $C$, но продадем на нулева цена, а горната граница е неопределена, стига стоката да е продаваема.

$k_{P}$ действително е еквивалентен на рентабилността на база приходи/цена.

Нека при така определената цена от формула (1) да определим печалбата, но като процент $k_{C}$ от разходите $C$, което също е еквивалентно на рентабилността на база разходи.

$$
\begin{aligned}
& \frac{C}{\left(1-\frac{k_{P}}{100}\right)}=P=C+\frac{k_{C}}{100} \cdot C \Rightarrow \\
& \Rightarrow 1=\left(1-\frac{k_{P}}{100}\right) \cdot\left(1+\frac{k_{C}}{100}\right) \Rightarrow \\
& \Rightarrow 1=1-\frac{k_{P}}{100}+\left(1-\frac{k_{P}}{100}\right) \cdot \frac{k_{C}}{100} \Rightarrow \\
& k_{P}=k_{C} \cdot\left(1-\frac{k_{P}}{100}\right) \\
& k_{C}=\frac{k_{P}}{1-\frac{k_{P}}{100}}
\end{aligned}
$$


което дава как се изразява процентът върху разходите, така че да се получи $k_{P} \%$ печалба. От (3) е ясно, че $k_{C}>k_{P}$. Можем да изразим и $k_{P}$ чрез $k_{C}$. От (2) следва

$$
\begin{aligned}
& k_{P}=k_{C}-\frac{k_{P} k_{C}}{100} \Rightarrow \\
& \Rightarrow k_{P} \cdot\left(1+\frac{k_{C}}{100}\right)=k_{C} \Rightarrow \\
& \Rightarrow k_{P}=\frac{k_{C}}{1+\frac{k_{C}}{100}} .
\end{aligned}
$$

От (4) също се вижда, че $k_{P}<k_{C}$.

Ако се върнем към разгледания пример:

$$
\begin{aligned}
& k_{P}=60 \% \Rightarrow \text { От (3) } \\
& k_{C}=\frac{60}{1-\frac{60}{100}}=\frac{60}{0,4}=\frac{600}{4}=150 \%
\end{aligned}
$$

и действително $10+\frac{150}{100} \cdot 10=10+15=25$ лв.

Основна цел на този подход според Иванов (Иванов, 2016, стр. 143) е да отразява вътрешно фирмените условия, свързани с разходите за производство и реализация на продукцията. Прилага се т.нар. „Прогресивна калкулация“ - от разходи към цена.

В края на тази първа част като извод, който можем да направим е: Процентьт печалба е най-реално да бъде върху общия приход. На тази основа може да се види какъв е делът на общите разходи и дельт на печалбата в цената.

\section{2. Модел за адекватно определяне на цената и общите разходи при изменение на част от разходите}

Нека въведем следните означения:

$P$ - цена;

$C$ - общи разходи;

$P_{f}$ - печалба;

$C_{i}$ - съставни разходи на общите разходи $(i=1 \div k)$.

Тогава

$$
P=C+P_{f}
$$

като

$$
C=\sum_{i=1}^{k} C_{i}
$$

и нека разходите $C_{i}$ са $l_{i} \%$ от общите разходи $C$, т.е. $C_{i}=\frac{l_{i}}{100} . C$ и $\sum_{i=1}^{k} l_{i}=100$ и нека в даден момент разходите $C_{i}$ се изменят с $p_{i} \%$. Тогава новите разходи $C_{i}^{\prime}=\left(1+\frac{p_{i}}{100}\right) \cdot C_{i}$, т.е. коефициентът на изменение на разходите $C_{i}$ е $k_{i}$, където $k_{i}=1+\frac{p_{i}}{100}$. (Тук искаме да подчертаем, че $p_{i}$ може и да е отрицателно, т.е. $\left.k_{i}<1\right)$ или 


$$
\begin{aligned}
C_{i}^{\prime} & =k_{i} \cdot C_{\text {и и }} \\
C^{\prime} & =\sum_{i=1}^{k} k_{i} \cdot C_{i}=\frac{C}{100} \cdot \sum_{i=1}^{k} k_{i} \cdot l_{i} .
\end{aligned}
$$

Искаме да пресметнем процента $p$ на изменение на общите разходи:

$$
\begin{aligned}
& p=\frac{C^{\prime}-C}{C} \cdot 100=\frac{\sum_{i=1}^{k} k_{i} \cdot C_{i}-\sum_{i=1}^{k} C_{i}}{C} \cdot 100 \\
& p=\frac{\sum_{i=1}^{k}\left(k_{i}-1\right) \cdot C_{i}}{C} \cdot 100=\sum_{i=1}^{k}\left(k_{i}-1\right) \cdot l_{i}
\end{aligned}
$$

Нека например само една от компонентите $C_{i}$ тьрпи изменение в даден момент и нека това е $C_{t}$ ( $t$ е конкретно цяло число в интервала $\left.[1 ; k]\right)$, а останалите разходи се запазват, т.е.

$$
\begin{aligned}
& C_{i}^{\prime}=C_{i}(i=1,2, \ldots, t-1, t+1, \ldots, k) \\
& \text { и } C_{t}^{\prime}=k_{t} C_{t}\left(k_{1}=k_{2}=\ldots=k_{t-1}=k_{t+1}=\ldots=k_{k}=1\right) .
\end{aligned}
$$

Тогава

$$
p=\frac{k_{t} C_{t}}{C} \cdot 100=\frac{p_{t} \cdot C_{t}}{C}=\frac{p_{t} \cdot l_{t}}{100}
$$

и от това, че $l_{t} \leq 100$, то от (7) се вижда, че $\frac{p_{t} l_{t}}{100}<p_{t}$, при $p_{t}>0$, т.е. увеличеният процент на общите разходи е по-малък от процента на увеличението на разходите $C_{t}$ и са равни само ако $C \equiv C_{t}$.

Нека се върнем към примера в началото.

Разходите за електроенергия са $10 \%$, т.е. $l_{\text {eл. }}=10$ и дори и да предположим, че има увеличение три пъти на електроенергията, то $p_{\text {ел. }}=200 \%$ и $p=\frac{200.10}{100}=20 \%$, т.е. общите разходи се увеличават с $20 \%$.

Ако има увеличение на повече от една компоненти $C_{i}$, то формула (7) придобива вида

$$
p=\frac{\sum_{i=1}^{k} p_{i} l_{i}}{100},
$$

и се вижда отново, че $p<p_{i}$, за всяко $p_{i}>0$, т.е. увеличението на общите разходи е помалко от увеличението на които и да е от съставящите го разходи.

Във формула (6) се забелязва, че процентът $p$ на изменение на общите разходи е средна претеглена на процентите на изменение на съставящите ги разходи $\left(p_{i}\right)$ с тегла коефициентите им на участие в общите разходи $\left(\frac{l_{i}}{100}\right)$.

Ако някои разходи тьрпят увеличение, други намаление, то за $p$ може да се получи както отрицателна, така и положителна стойност, а това зависи както от стойностите на 
самите $p_{i}$, така и от процентното участие на разходите $C_{i}$ в общите разходи $C$. В този аспект за по-голяма конкретика тук ще формулираме и докажем следното:

Твърдение: Нека $C=\sum_{i=1}^{n} C_{i}$ и $C_{i}=\frac{l_{i}}{100} \cdot C\left(\sum_{i=1}^{n} l_{i}=1\right)$ и нека първите $k(k<n)$ вида разходи се увеличават с $p_{i} \%(i=1 \div k)$, а останалите $n-k$ вида разходи се намалят $p_{i} \%(i=k+1 \div n)$. Тогава, ако общите разходи се изменят с $p \%$ то:

1) Ако $p>0$, то $p<\max _{i \in[1 ; k]}\left\{p_{i}\right\}$.

2) Ако $p<0$, то $p>-\max _{i \in[k+1 ; n]}\left\{p_{i}\right\}$.

Доказателство:

1. $p=\frac{\sum_{i=1}^{n} p_{i} l_{i}}{100}=\frac{\sum_{i=1}^{k} p_{i} l_{i}-\sum_{i=k+1}^{n} p_{i} l_{i}}{100}<\frac{\max _{i \in[1 ; k]}\left\{p_{i}\right\} \sum l_{i}-\sum_{i=k+1}^{n} p_{i} l_{i}}{100}<$ $<\frac{100 \cdot \max _{i \in[1 ; k]}\left\{p_{i}\right\}-\stackrel{>}{>} \overbrace{}^{-0}}{100}<\max _{i \in[1 ; k]}\left\{p_{i}\right\}$.

2. $p=\frac{\sum_{i=1}^{k} p_{i} l_{i}-\sum_{i=k+1}^{n} p_{i} l_{i}}{100}>\frac{-\sum_{i=k+1}^{n} p_{i} l_{i}}{100}>\frac{-\max _{i \in[k+1 ; n]}\left\{p_{i}\right\} \sum_{i=k+1}^{n} l_{i}}{100}>$

$$
>\frac{-\max _{i \in[k+1 ; n]}\left\{p_{i}\right\} .100}{100}=-\max _{i \in[k+1 ; n]}\left\{p_{i}\right\} .
$$

Извод: Процентът на изменение на разходите винаги е между най-големия процент на увеличение на някои от разходите и между отрицателната стойност на най-големия процент на намаление на някои от разходите.

Нека сега разгледаме какво е адекватното изменение на цената на стоката. Да се върнем към формула (5) $P=C+P_{f}$ и печалбата $P_{f}$ я разгледаме чрез $K_{C}$ (макар че изказах, своето мнение за процента печалба, че е по-адекватен ако се изчислява на база рентабилност относно приходите, т.е. $k_{P}$, но във формули (3) и (4) бе изразена връзката между $k_{P}$ и $k_{C}$ ).

Тогава:

$$
P=C+\frac{k_{C}}{100} . C
$$

Припомняме, че $k_{C}$ e \% от разходите за определяне на печалбата.

Първи вариант: Нека в абсолютни единици печалбата се запазва същата, т.е.

$$
\begin{aligned}
& P_{f}^{\prime}=P_{f}=\frac{k_{C}}{100} . C, \\
& P^{\prime}=C^{\prime}+\frac{k_{C}}{100} . C .
\end{aligned}
$$

Процентьт $p_{P}$ на изменение на цената е: 


$$
\begin{aligned}
& p_{P}=\frac{P^{\prime}-P}{P} \cdot 100=\frac{C^{\prime}-C}{C\left(1+\frac{k_{C}}{100}\right)} \cdot 100=\frac{\sum_{i=1}^{k} \frac{p_{i}}{100} \cdot C_{i}}{C\left(1+\frac{k_{C}}{100}\right)} \cdot 100= \\
& =\frac{\sum_{i=1}^{k} p_{i} l_{i} C}{C\left(1+\frac{k_{C}}{100}\right)}=\frac{\sum_{i=1}^{k} p_{i} l_{i}}{100\left(1+\frac{k_{C}}{100}\right)}
\end{aligned}
$$

Ако се върнем отново към примера в началото:

$l_{\text {ел. }}=10 \%, p_{\text {ел. }}=200 \%$ и нека $k_{C}=30 \%$, т.е. общите разходи са 1 лв., от които 0,10 лв. за електроенергия и 0,90 лв. други разходи и $\frac{30}{100} \cdot 1=0,30$ лв. печалба и тогава $P=1+0,30=1,30$ лв.

От (8): $p_{P}=\frac{p_{e л} l_{e л}}{100\left(1+\frac{K_{C}}{100}\right)}=\frac{200 \cdot 10}{100.1,3}=\frac{200}{13} \approx 15,4 \%$.

Тогава новата адекватна цена трябва да бъде $P^{\prime}=1,154 . P=1,154.1,3=1,50$ лв., а не 1,70 лв.

Във формула (8) можем също така да забележим, че множителят $\frac{\sum_{i=1}^{k} p_{i} l_{i}}{100}$ e $p$ (процента на увеличение на общите разходи) от (7') или

$$
p_{P}=\frac{p}{\left(1+\frac{k_{C}}{100}\right)}
$$

Ясно се вижда и, че:

$$
p_{P}<p<p_{t}, \text { при } p_{t}>0 \text {. }
$$

За примера в началото преди малко изчислихме, че $p=20 \%$ и ако се използва формула (8') $p_{P}=\frac{20}{1,3} \approx 15,4 \%$, т.е. получава се същият резултат.

Втори вариант: Нека търпи промяна и печалбата, т.е. тя също се изменя със същия процент $k_{C}$, но върху новите разходи, или

$$
P^{\prime}=C^{\prime}+\frac{k_{C}}{100} \cdot C^{\prime}=\left(1+\frac{k_{C}}{100}\right) \cdot C^{\prime}
$$

Тогава

$$
p_{P}=\frac{\left(1+\frac{k_{C}}{100}\right) \cdot C^{\prime}-\left(1+\frac{k_{C}}{100}\right) \cdot C}{\left(1+\frac{k_{C}}{100}\right) \cdot C} \cdot 100=\frac{C^{\prime}-C}{C} \cdot 100=p,
$$


или процентьт на изменение на цената е същият като процентът на изменение на разходите и $p_{P}=p<p_{t}, p_{t}>0$.

Ако се върнем отново към първоначалния пример, но при този втори вариант, то за цената се получава $P^{\prime}=1,2.1,3=1,56$ лв., но отново не е $1,70 л в$.

Преди да завършим нека разгледаме един пример, свързан с някои теоретични постановки, които бяха изложени.

Нека разходите $C$ имат три компонента: $C_{1}, C_{2}$ и $C_{3}$, като $l_{1}=40 \%, l_{2}=25 \%$ и $l_{3}=35 \%$ и нека $p_{1}=-10 \%, p_{2}=15 \%$ и $p_{3}=5 \%$.

Тогава $p=\frac{p_{1} l_{1}+p_{2} l_{2}+p_{3} l_{3}}{100}$ (по формула $\left.\left(7^{\prime}\right)\right)$ и

$$
p=\frac{40 .(-10)+25.15+5.35}{100}=1,5 \%
$$

a $p_{P}=\frac{p}{1+\frac{k_{C}}{100}}$ и ако $k_{C}=80 \%$, то $p_{P}=\frac{1,5}{1,8} \approx 0,83 \%$. Но ако изменим само $p_{1}$ на $-15 \%$, то за $p$ се получава $-0,5 \%$, а за $p_{P} \approx-0,28 \%$.

\section{Заключение}

Като обобщение на изложеното можем да направим следните основни изводи:

1. По наше мнение по-целесъобразно е печалбата в процентно изражение да се изчислява на основа приходи.

2. При увеличение на някои от разходите с $p_{t} \%$ и на тази основа увеличението на разходите е $p$, а на печалбата $p_{P}$, то винаги е в сила $p_{t} \geq p \geq p_{P}$, като $p_{t}=p$, ако $C \equiv C_{t}$ и $p=p_{P}$, ако печалбата в абсолютни единици също се увеличава със същия досегашен процент, но върху новите разходи.

3. Отново по наше мнение, считаме, че е целесъобразно в новата цена да бъде отразена само промяната в разходите и печалбата в абсолютни единици да се запази същата. Това не би довело до промяна на пазарния дял на производителя и би се запазила неговата конкурентоспособност.

4. За нивото на конкурентоспособността би спомогнало и това, че тези които формират ценовите равнища на своите продукти/услуги е желателно да се интересуват и използват научнообоснованите методи за адекватно ценообразуване, а не да го правят по усет и първоначален инстинкт.

\section{References}

1. Blagoev, B. i dr. (2003) Upravlenie na tsenoobrazuvaneto. Varna: Nauka i ikonomika.

2. Vladimirova, Y. (2010) Tseni i tsenoobrazuvane. Sofiya: Stopanstvo.

3. Ivanov, Y. (2019) Tsenova strategiya za balgarskite akumulatorni zavodi. Varna: Dangrafik.

4. Ivanov, Y. (2016) Tsenovi aspekti na konkurentosposobnostta. Monografichna biblioteka „Prof. Tsani Kalyandzhiev“, Varna: Nauka i ikonomika.

5. Lilova, R. (2012) Tsenoviyat menidzhmant - vazmozhnosti za optimizirane na finansovite rezultati na firmata. Biblioteka „Obrazovanie i nauka“, knizhka 22, SA „D.A.Tsenov“, Svishtov.

6. Lipsits, I. V. (2005) Tsenoobrazovanie - upravlenie tsenoobrazovaniem v organizatsii. Moskva: Ekonomisty.

7. Monev, P. (2002) Tsenoobrazuvane v stroitelstvoto. Varna: Nauka i ikonomika. 
8. Netseva-Porcheva, T. (2012) Tsenoobrazuvane na baza tsennost. V tarsene na pechelivshi tsenovi reshenie. Sofiya: Stopanstvo.

9. Nagle, T., J.Hogan, J.Zale (2011) The Strategy and Tactics Pricing - A Guide to Growing More Profitable. 5th Ed. Pearson.

10. Maxwell, S. (2008) The Price is wrong. Understanding what makes a price seem fair and the true of unfair Pricing. Jon Wily \& Sons inc. 